\section{More Than One Ever Wanted To Know About X-Ray Detectors Part VIII: If I Know It's There, Why Can't I See It?}

D. Clark Turner, Ph.D, MOXTEK, Inc.

This month we are going to deviate from Mark Lund's normal discussion and talk about one of the practical problems that must be considered when using an x-ray detector on a scanning electron microscope to solve real-world problems.

Several years ago I worked in the analytical laboratory of a major semiconductor manufacturer. One day a process engineer came into the lab and asked us to develop an analytical method to determine the amount of copper in an aluminum film on a silicon wafer. The levels of copper would be somewhere between $0.5 \%$ and $1.0 \%$. He gave me a sample, saying that he didn't

\section{Engineering Opportunities}

Continued growth at FEI Company, the world leader in commercial applications of Focused lon Beam Technology, has resulted in several immediate openings for engineers throughout the company.

\section{EE Production Engineer}

We need an EE Production Engineer to join or manufacturing group. Re quires a BSEE with $3-5$ years experience in a manufacturing environment including self-directed work teams. Must be familiar with PC controlled systems, digital electronics, NPI process and state-of-the-art teamorientated manufacturing processs. Any ISO 9000 experience a plus.

\section{Applications Engineer}

We need an Application Engineer to join our technical marketing group and be responsible for applications development, performing sample work and operating systems for potential customers. Requires a BS/MS with 3-5 years' direct experience operating TEM, SEM or similar analytical laboratory equipment. Should be familiar with $I C$ manufacturing inspection process.

\section{Digital EE}

We need a Digital EE to join our engineering group. Requires a BSEE or equivalent, 5 years experience with digital circuit design in a team-oriented research environment, and familiarity with logic analyzers, oscilloscopes, etc. Must have experience in DOS, Windows, C, and MS Basic, familiarity with HW/SW aspects of $x 86$ backplane including Pentium, experience in PALS, FPBAs, $x 86$ processors, $A / D+D / A$, embedded processors, and interface protocols, and NPI experience.

In addition to the listed technical and educational qualifications, the applicant selected for each of these positions must be eligible for a passport since some training in Europe is required

FEl offers an outstanding compensation/benefits package including profit share, $401(\mathrm{~K})$ with match, stock options, fully paid medical/dental for em ployees and family, vacation/sick time/personal time, tuition reimbursement, athletic club subsidy, and the opportunity to work closely with some of the world's leading scientists and engineers in this industry.

Interested principals please contact our HR Department at (503)640-7500. fax: (503)640-7509 or eMail: MES@FEICO.COM - or send your resume to FEI Company, 7451 N.E. Evergreen Parkway, Hillsboro, OR 97124.

$$
\text { FEl Company is an Equal Opportunity Employer. }
$$

know the exact concentration of copper but was certain that it was at least $0.5 \%$. Well, being smart analytical chemists we determined that we wanted to do as little sample preparation as possible to get the analysis (we were a little bit lazy, too, but who isn't?). We fractured the wafer and mounted a small piece for analysis using the EDS $x$-ray attachment on our SEM. We knew this wouldn't be a quantitative analysis, but just wanted to make sure there would be adequate signal for quantification later. Much to our chagrin there was almost no copper signal distinguishable above background in the $x$-ray spectrum that we collected. This was a particularly disconcerting result because we'd promised the engineer we'd have a method developed within the week, and now we were going to have to do some real work! Why couldn't we see the copper in the spectrum?

First you must understand that in order to say conclusively that there is a peak in the x-ray spectrum, the peak must be statistically "above background" $X$-ray events occur in a Poisson distribution, which was discussed by Mark in Part 2 of this series. The standard deviation of this distribution is the square root of the counts. In order to say with $98 \%$ certainty that a peak is real, the peak height must exceed the height of the background by 2 (background) ${ }^{1 / 2}$. If the x-ray counts in a peak exceed the background by 3 (background) ${ }^{1 / 2}$ there is nearly a $99.9 \%$ certainty that the peak exists. Since we couldn't see the peak conclusively we either had too much background or not enough peak, or both

Let's look at the background side first. If there is no spectral overlap of characteristic lines and no artifacts in the spectrum, then the main contribution to the background near the $x$-ray peak is broadband bremsstrahlung. (I am told that bremsstrahlung is German for "braking radiation".) Bremsstrahlung radiation is produced whenever an electric charge changes speed or direction. As SEM beam electrons interact in the sample, they can approach near enough to the nucleus of a sample atom to be deflected by its positive charge. The resulting change in direction is an acceleration of the electron, and an $x$-ray photon is emitted. This process can occur numerous times until the electron loses all of its kinetic energy. Occasionally, an electron is brought to rest in a single collision, and its entire kinetic energy is converted into a single photon of equivalent energy. So the maximum energy of the broadband bremsstrahlung is equal to the accelerating potential of the SEM, and the shape of the background continuum is determined both by the accelerating voltage and by the sample material.

Unfortunately, since the production of bremsstrahlung is fundamental to the physics of the electron-matter interaction, there are very few remedies for reducing the background. One way to reduce the bremsstrahlung is to reduce the amount of matter for electrons interaction. Rather than placing the sample on a typical SEM stub, which is infinitely thick to electrons, the sample can be placed on a thin polymer film. If the polymer is sufficiently thin then many of the electrons will pass through without losing large amounts of kinetic energy to bremsstrahlung, significantly reducing the $x$-ray background. In our laboratory we are currently performing experiments to demonstrate the improvement in sensitivity using this technique and hope to publish our results during the next year

Now that we've looked at background, what about the size of the peak? When an electron beam is incident on a sample it interacts not just at the surface but within an interaction volume. This volume is shaped somewhat like a pear -narrow at the top and spherically shaped at the bottom. It turns out that the depth range of $20 \mathrm{KV}$ electrons in silicon or aluminum is about 3 microns, with a similar lateral distance being excited. Since the thickness of the aluminum film we were investigating was less than 1 micron, the majority of the volume being analyzed was the silicon wafer rather than the aluminum film. This hurts us in two ways. First, rather than being $0.5 \%$ in concentration, the copper atoms are now maybe only $0.05 \%$ or less of the total atoms in the excitation volume. Second, we now have a significant number of silicon $x$-rays in the spectrum. Since the detector can only process one $x$-ray at a time, as we tie it up processing silicon $x$-rays it is not available to process copper $x$-rays.

There are at least three ways to get around these problems. Reducing the accelerating voltage on the SEM will reduce the penetration depth of the electrons and result in a smaller excitation volume. This increases the effective concentration of the copper. However, the accelerating potential must be kept above the $K$ shell absorption edge in order to produce $x$-rays, and should normally be at least twice this energy. For copper the $\mathrm{K}$ shell absorption edge is $8.98 \mathrm{KeV}$, so we would want to be at $18 \mathrm{KV}$ or above for the SEM accelerating potential. So this isn't a real good option for copper but is particularly effective for lighter elements. 
The alumınum film can also be made much thicker. In this way more of the excitation volume can be filled with aluminum and copper, and less with silicon. This is a good solution where practical, but in our case the engineers wanted to sample on actual production wafers and weren't willing to make the films $3 \mathrm{ml}$ crons thick.

Finally, you can collect the x-rays for a longer time. As long as the counts in the peak are accumulating faster than the square root of the background counts, eventually the peak will be distinguishable. It can be quite frustrating, however, to find yourself in the opposite condition where you have collected an $x$-ray spectrum for an hour or two and there is still no peak! Don't be deceived, though - just because you don't see a peak doesn't mean that the element is not there!

(By the way, for logistical reasons we finally established the analytical method to be $x$-ray fluorescence spectromety utilizing an energy-dispersive XRF instrument that was already located on the production floor near the aluminum evaporators.)

\section{Budget Constraints?}

\$ Lease or rent your new SEM, TEM, EDS or other laboratory equipment with buyout options.

\$Plans are easily tailored to your budgetary needs.

\$Our fast approval, low rates, and pleasant service will please you.

\section{For more information call: \\ Greg Smith at (800)700-6680 \\ Concord Funding Group 26 Main Street \\ Concord, MA 01742}

An Effective, Cost-Free Holder/Desiccator for Storage of SEM Stubs

Glenn Walker and Jerry Hartenburg, Eastern Michigan University, and Chris Edwards, University of Michigan

Two of the aggravations of effectively managing an EM unit are cost containment and effective storage of large numbers of specimens. For the past several years we have enjoyed the benefits of a stub-storage box (for pin-type stubs) available to microscopists without cost. These boxes, which held micro-pipette tips, are regularly discarded by molecular biology and biochemistry labs. In fact, since colleagues have recognized that we use their spent plastic boxes, we have been inundated with boxes oniginally slated for recycling. While several types of these boxes are available, most measure approximately twelve $\mathrm{cm}$ by eight $\mathrm{cm}$ and have a plastic platform with ninetysix holes. The boxes hold eight one-inch stubs and six half-inch stubs or thirtytwo half-inch stubs. These contrast with commercially available specimen mount holders which accommodate either four or twelve half-inch stubs and cost at least a couple of dollars each. We find, since availability of the holders is not limiting, that the boxes which are fitted with a removable bottom are preferable to those with a solidly molded casing. We gain easy access to the bottom of these boxes and either insert a packet of 'Drierite' wrapped in tissue to prevent the escape of dust or pour in silicon desiccation beads. Unlike the commercially available holders, these boxes provide a desiccated environment, are available at no cost and accommodate a large number of specimen holders. Our students and faculty are no longer inconvenienced by a variety of stub-holding options such as drilling holes in wood or punching holes in cardboard and storing these holders in assorted containers with a desiccant. Further, specimen shelving allows large numbers of stubs to be easily and efficiently stored in these like-sized boxes.

\section{h
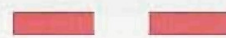

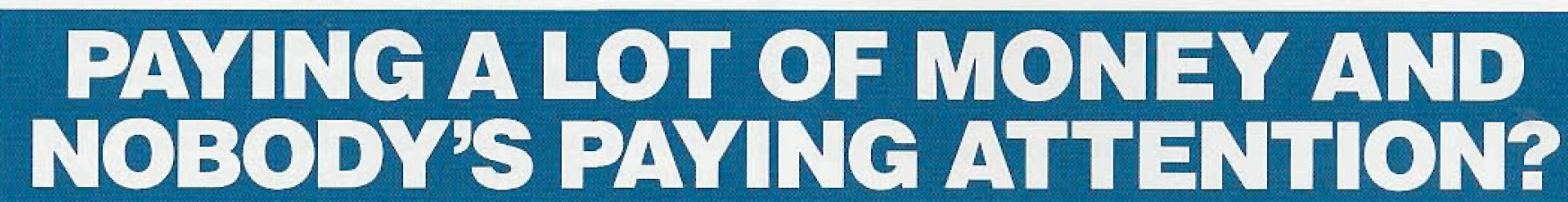

Service Contracts are expensive. Are you getting the service you paid for? Call the Materials Analytical Services' EM SERVICE GROUP before you sign another Service Contract.

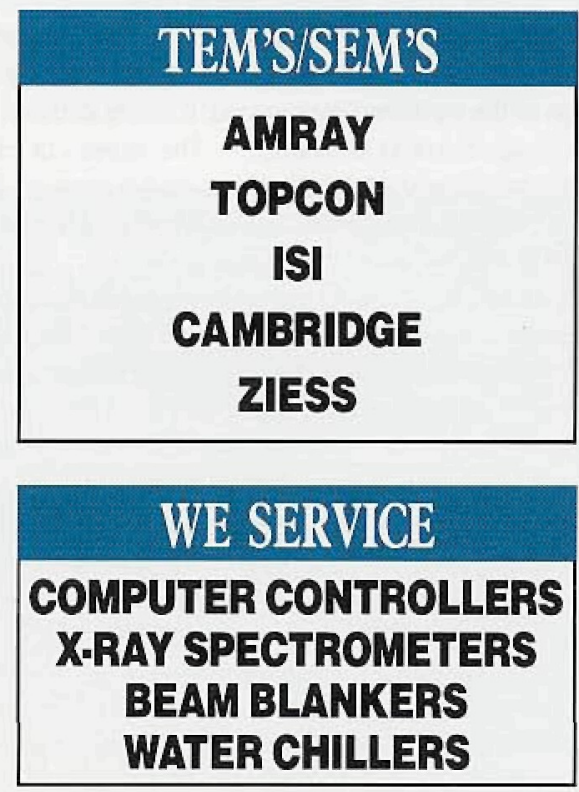

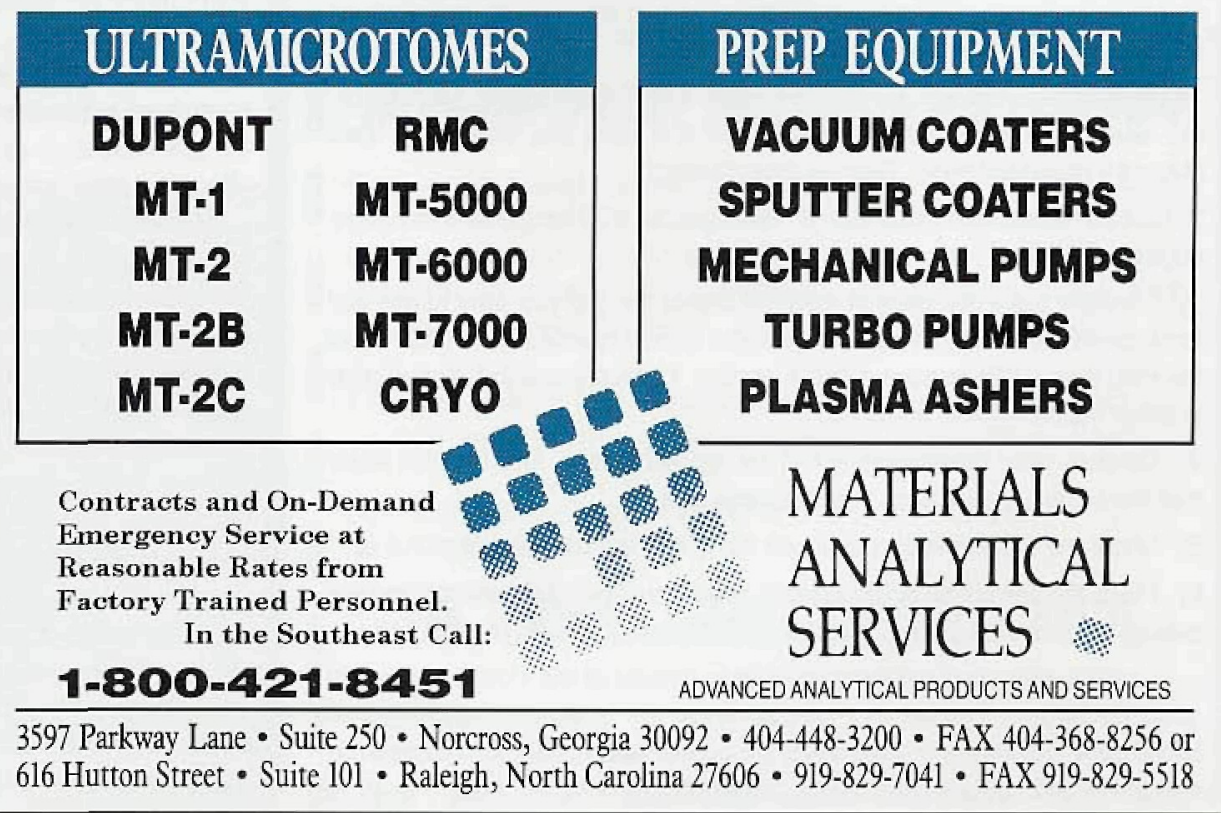

